observation which at first sight seemed to show the existence of residual diamagnetic polarity in a diamagnetic substance after exposure to a strong field, and remarks that this seemed an incomprehensible result. It appears to me that this result, should it be confirmed, is not incomprehensible on Weber's theory of diamagnetism, if we supplement that by a modification of the Ampere-Weber theory of ordinary magnetisation.

Suppose that the induced currents in the molecules of a diamagnetic substance are confined to definite channels, that there is little or no primitive current, and that the molecules are capable of being deflected. Then as the field is increased, each molecule is turned so that the plane of its channel becomes more and more nearly parallel to the lines of force. We may assume that this turning of the molecules is resisted, like the turning of the molecules of iron, and that when the field is withdrawn they return more or less completely towards their initial positions.

Experiments with iron and steel show that in the turning of the molecules the resistance while the field is being applied is on the whole greater than the restoring force, while the field is being removed: in fact something very like static friction acts on each molecule. There is what I have elsewhere called "hysteresis," or lagging behind, in the relation of the molecule's movement to the magnetising force. If this molecular quasi-friction also exists in diamagnetic substances, and if the molecular channels are turned at all, they will, during the removal of the field, be in less favourable positions for the induction of currents than they were in during the application of the field. There will consequently be a residue of current in each when the field is wholly withdrawn; and these residues will make the substance a permanent "diamagnet."

But the fact that this result would be comprehensible is no evidence of its truth, and apparently Dr. Lodge inclines to interpret the experiment referred to in an entirely differentindeed opposite-sense. Moreover, his other results show not residual diamagnetism, but residual paramagnetism in diamagnetic substances which have been immersed in a very strong field.

Now I think this result may also be interpreted in terms of the magnetic theory of magnetisation ; and the purpose of this communication is to suggest an explanation which seems to me so probable that it may perhaps serve, until Dr. Lodge confirms these results, as a set-off against the suspicion he has cast on them by suggesting the presence of iron in his diamagnetic bodies.

When we begin to magnetise iron by a field which increases from zero, we find at first scarcely a trace of magnetisation. A curve showing the relation of intensity of magnetism to magnetising torce starts off (as nearly as can be judged) tangent to the line along which the magnetising force is plotted, but soon, of course, takes ia rapid bend as the permeability increases. This is very consistent with the idea that the molecular electromagnets are held back from turning by a sort of static friction which requires the field to reach a finite value (different perhaps for different molecules) before the process of turning begins. But what has happened before this process begins? Diamagnetic induction has been going on in each molecule that has not begun to turn; and hence, if the molecular configuration is rigid for a magnetising force of any finite magnitude, the substance is diamagnetic in that and all weaker fields.

If this be the case in iron (and the experimental evidence certainly points to the existence of a finite frictional resistance to the turning of the molecules) that metal is really diamagnetic in excessively weak fields, because the molecules are fixed by friction ; then very paramagnetic in stronger fields, because the molecules are turning; and, finally, diamagnetic in a field strong enough to turn the molecules as far as they will go, and to induce currents in them which swamp the primitive Amperian currents.

Next, imagine a substance whose molecules are held by friction in a very tight grip, so that no moderate magnetising force is able to alter their configuration. The substance is then diamagnetic, and when the field is withdrawn there is no residual polarity. But let a field be applied strong enough to begin turning the molecules. This will cause a decrease of diamarnetic susceptibility. And when the field is withdrawn the molecules remain deflected, and the substance is a permanent paramagnet.

Now this is exactly what Dr. Lodge has observed in his copper, coke, wood, and so forth. They behaved as diamag- netics while in the field, but showed paramagnetic polarity when withdrawn from it.

My suggestion, then, is that in diamagnetics, as in paramagnetics, there are strong primitive Ampèrian currents circulating in the molecular channels. That in a strongly paramagnetic substance such as iron there is comparatively little molecular rigidity, so that the molecules begin to turn even in very weak fields; the induction of currents in their channels then plays a very insignificant part in the magnetisation. That in a diamagnetic substance, on the other hand, the molecules stick so fast that in any moderate field they have scarcely begun to turn ; the induction of currents goes on independently of the existence of the primitive currents, and is then practically the whole affair. But if the field be made strong enough the molecules begin to turn, not in the way spoken of in the earlier part of this communication (where it was assumed that the induced currents swamped the primitive currents), but in the way in which the molecules of iron turn. Then common magnetisation becomes superposed on diamagnetic induction. And when the field is withdrawn the molecules are left with a paramagnetic alignment, and with their primitive Amperian currents strengthened, if anything, since they have been facing more favourably during the withdrawal of the field.

There is nothing to show that the primitive Amperian currents are not as strong and as numerous in copper or bismuth as in iron. If they are, and if we could only apply a field strong enough to force them into alignment, we might expect to find, in substances so hard to magnetise, a permanence in the residual magnetism which would put steel to the blush.

University College, Dundee, March 27 J. A. EwInG

\section{Ferocity of Rats}

I HAVE recently had occasion to chloroform a number of wild rats for the purpose of procuring their blood. The rats are sent to me by a ratcatcher, who places from six to twelve in the same trap or cage. It usually happens that, within a few hours after their imprisonment, some of their number are killed and eaten by the others; while they all exhibit scars as the result of their struggle for existence in confined quarters.

A few days ago I placed two wild rats in a cage, and for a long time endeavoured unsuccessfully to catch the larger one under a bell-jar let in through a doorway in the top of the cage. The rat perfectly well understood my object, and for about ten minutes succeeded by his agility in thwarting it. This animal, therefore, must have been in as great a state of alarm as it is possible that a rat could be. Nevertheless, after the ten minutes' chase inside the cage-during which he had been many times very nearly caught - he appeared to be suddenly seized with a violent outburst of ferocity against his fellow-prisoner; for he fell upon the smaller rat, drove it into a corner of the cage, and killed it by biting its throat. By means of a glass rod I drove him away, drew the dead body of his victim beneath the doorway in the roof of the cage, and held the bottom of the bell-jar just above the dead rat. I had not long to wait before the living one again fell upon his victim and began to devour the carcass. It was then an easy matter to lower the bell-jar over both the living and the dead, when, by pouring chloroform in at the open top of the bell-jar, I quickly reduced the murderer to a state of insensibility. But up to the very last moment of consciousness this animal continued to bury his fangs in the body of the little rat, and even after his head had dropped away in stupor the jaws still continued to move as if he were enjoying the feast in his dreams.

Now, I do not believe that any instance of ferocity at all approaching this could be found in any other animal. But it has been suggested to me that the fact may have been due to a kind of cmotional insanity produced by extreme terror. I therefore write to ask whether any of your readers can supply me with additional facts bearing upon the subject. In particular, is it the habit of wild rats when not confined, or when in a state of nature, to devour one another? Or do they only do so when shut up together in a cage? GeORGE J. Romanes

\section{The Recent Weather}

THE enclosed extract from the log of one of the "excellent" observers for the Meteorological Office may be interesting to some of your readers, as bearing upon the large amount of 